THE AstrophysiCAL JouRNAL, 369:L31-L34, 1991 March 10

(C) 1991. The American Astronomical Society. All rights reserved. Printed in U.S.A.

\title{
NGC 1068: RESOLUTION OF NUCLEAR STRUCTURE IN THE OPTICAL CONTINUUM
}

\author{
Roger Lynds, ${ }^{1}$ S. M. FAber, ${ }^{2}$ Edward J. Groth, ${ }^{3}$ Jon A. Holtzman, ${ }^{4}$ Robert M. Light, ${ }^{2}$ \\ Earl J. O’Neil, Jr., ${ }^{1}$ William A. Baum, ${ }^{5}$ Douglas G. Currie, ${ }^{6}$ S. P. Ewald, ${ }^{7}$ \\ J. Jeff Hester, ${ }^{8}$ Jerome A. Kristian, ${ }^{9}$ P. Kenneth Seidelmann, ${ }^{10}$ \\ EDWARD J. ShaYA, ${ }^{6}$ AND JAMES A. WeStPhaL ${ }^{11}$ \\ Received 1990 October 18 ; accepted 1990 November 27
}

\begin{abstract}
An optical continuum image of the Seyfert galaxy NGC 1068 has been obtained with the Planetary Camera on the Hubble Space Telescope as part of the SAO/ERO program. The filter used, F547M, has an effective wavelength and a width of approximately 5470 and $650 \AA$ and excludes all discrete emission lines that might be present. The point-spread function, although compromised by the aberrations of the telescope optics, does permit significant response at the 0 .'1 resolution originally anticipated.

Deconvolution of the image for the effects of the point-spread function reveals a bright nucleus embedded in an irregular cloudlike structure which is well differentiated against the background of the galaxy. The nucleus is resolved, with a FWHM of $\sim 0$ " 15 , or $11 \mathrm{pc}$ and there is no evidence for any unresolved nuclear component. The lack of an unresolved point source is consistent with the picture in which the broad-line region is seen indirectly via electron scattering, but the precise geometry of the scattering region cannot yet be inferred. The cloud surrounding the nucleus is elongated in the NE-SW direction and has extreme dimensions of 3 ..5 $\times 1$.7 $(255 \times 124 \mathrm{pc})$. Although the extent of the cloud is roughly centered on the nucleus, its brightness centroid is situated 0".4 southwest of the nucleus. Because of the relative photometric dominance of the cloud with respect to both the nucleus and the underlying galaxy and the corresponding dominance of unpolarized star light found by Antonucci and Miller, the conclusion seems inescapable that the light from the cloud is contributed largely by stars. But in appearance the cloud is distinctly dissimilar to our expectation for either young or old stellar systems, and it seems to bear no simple relationship to the numerous features that have heretofore been resolved at other optical, infrared, and radio wavelengths. A determination of the true nature of both the cloud and the nuclear feature through spectroscopic and polarimetric measurements is of the highest priority.

Subject headings: galaxies: individual (NGC 1068) - galaxies: Seyfert
\end{abstract}

\section{INTRODUCTION}

NGC 1068 has been the subject of numerous investigations and theoretical interpretations for more than 20 years. Here we confine our attention to a few of the discoveries of discrete features within the central few arcseconds. First of all, there is the spectropolarimetric investigation of Antonucci \& Miller (1985), which established that within a 2".8 circular aperture centered on the nucleus both the nonstellar continuum and the broad emission lines are strongly and about equally polarized with little wavelength dependence. This led to the view that the source for both components is hidden from direct observation and is seen only by scattering from electrons.

\footnotetext{
${ }^{1}$ Kitt Peak National Observatory, National Optical Astronomy Observatories, Tucson, AZ 85726. The National Optical Astronomy Observatories are operated by the Association of Universities for Research in Astronomy, Inc., under cooperative agreement with the National Science Foundation.

${ }^{2}$ UCO/Lick Observatories, Board of Studies in Astronomy and Astrophysics, University of California, Santa Cruz, CA 95064.

3 Physics Department, Princeton University, Princeton, NJ 08544.

${ }^{4}$ Lowell Observatory, Flagstaff, AZ 86001.

${ }^{5}$ Astronomy Department, University of Washington, Seattle, WA 98105.

${ }^{6}$ Department of Physics and Astronomy, University of Maryland College Park, MD 20742.

${ }^{7}$ Space Telescope Science Institute, Baltimore, MD 21218.

${ }^{8}$ Infrared Processing and Analysis Center, California Institute of Technology, Pasadena, CA 91125.

9 Carnegie Institution of Washington, Pasadena, CA 91101.

${ }^{10}$ U.S. Naval Observatory, Washington, DC 20390.

${ }^{11}$ Division of Geological and Planetary Sciences, California Institute of Technology, Pasadena, CA 91125.
}

It has been known since the pioneering work of Walker (1968) that the nuclear region of NGC 1068 contains several discrete emission-line clouds showing high internal and differential motions with respect to the nucleus. Among the several emission-line investigations since that time are those attempting to resolve the discrete clouds by means of speckle interferometry. One by Ebstein, Carleton, \& Papaliolios (1989) in the light of [O III] $\lambda 5007$ shows the most consistency among diverse sets of observational data and reveals several discrete sources in addition to one which may arguably be called the nucleus. One speckle reconstruction for $5500 \AA$ continuum light shows mainly a central source elongated asymmetrically in the NE-SW direction.

Finally, the central radio source in NGC 1068, the strongest component of a larger jetlike structure elongated in the NE-SW direction, has been shown at centimeter wavelengths to be resolved into a "bent" triple source with an outercomponent separation of 0".65 (van der Hulst, Hummel, \& Dickey 1982; Wilson \& Ulvestad 1983; Ulvestad, Neff, \& Wilson 1987). The southern component is a little west of due south from the central component, and the northern component lies to the northeast. These features may have optical counterparts in the speckle reconstructions of Ebstein, Carleton, \& Papaliolios (1989).

\section{THE NEW OBSERVATIONS}

We report here on one $18 \mathrm{~s}$ exposure of NGC 1068 made with the $\mathrm{f} / 30$ Planetary Camera optics of the Wide-Field/ 
Planetary Camera on the Hubble Space Telescope (HST) through the filter F547M, which has an effective wavelength and a half-response width of approximately 5470 and $650 \AA$, respectively. (For a detailed description of the instrument, see $\rightarrow$ Griffiths 1990.) No discrete emission lines of significant strength are admitted by the bandpass. The scale in a Planetary Camera image is 0".043 per pixel. Assuming a distance modulus of 15.1 for NGC 1068 (for a Hubble constant of $H_{0}=$ $75 \mathrm{~km} \mathrm{~s}^{-1} \mathrm{Mpc}^{-1}$ ), $1^{\prime \prime}$ corresponds to $73.3 \mathrm{pc}$, and 1 pixel corresponds to $3.15 \mathrm{pc}$.

A $512 \times 512$ pixel submatrix of the recorded image of NGC 1068 is reproduced with logarithmic intensity scaling in Figure 1 (Plate L5). The arrow in the lower right-hand corner is $5^{\prime \prime}$ long and indicates the direction of north (11.5 left of vertical). The image has been subjected to cosmic-ray removal and correction for spatial response variations using our best flat-field calibration, which is not as reliable as we would like but is seemingly adequate for the present purpose. As documented elsewhere in this issue, the $H S T$ suffers from a large amount of optical aberration. Fortunately, the point-spread function (PSF) as revealed by star images show a sharp core, containing $\sim 15 \%$ of the energy, approaching in resolution the 0.1 value originally expected. Thus, one is able to see in Figure 1 fine structural detail, albeit at low contrast, and we have found that such images respond usefully to a degree of deconvolution.

Unfortunately, the determination of the PSF has thus far been pretty much a matter of luck, as no specific PSF exposures were made. In the present case we have drawn upon exposures of the 30 Doradus region made shortly before the exposure of NGC 1068 for star images adequate to characterize the PSF. Five separate images of a star $\sim 100$ pixels from the location of the nucleus of NGC 1068 have been averaged to form the PSF. Before averaging, the star images were shifted (resampled by Stirling third-order interpolation) to correct for registration defects as judged by centroiding on their peaks. No resampling was performed after averaging.

The origin of the deconvolution algorithm that has been applied to the NGC 1068 image is unknown to us. It is probably at least a century old and consists merely of seeking by iterative correction that model which when convolved with the PSF gives a result numerically equal to the original observation. This algorithm was used because it is very fast, it is entirely linear, it performs no operations of imponderable consequence, it introduces no constraints such as positivity, and it happens to have been recently implemented by one of the authors (Lynds 1991). The corrections are applied by addition, not multiplication, and are filtered by the Fourier transform modulus of the PSF so that the rate of convergence tends to be proportional to signal-to-noise ratio in the Fourier domain. Thus, the convergence tends to be most rapid at low spatial frequencies, and termination of iteration establishes the degree of convergence at high frequencies.

Among the limitations of the algorithm, it is incapable of recognizing or responding to spatial context in the object domain (not necessarily a fault). It is also well to remember that we are dealing with incrementally sampled (perhaps undersampled in our case) representations of both PSF and object, and deconvolutions of the type used here will be limited by that de facto sampling, especially in the presence of noise in both PSF and object. Finally, we know that the PSF is fielddependent, so that it is certain to be somewhat inappropriate anywhere in the field except near the point where it was derived. We generally adopt the view that a feature seen in the deconvolution is not real unless it can be seen in the original image and that we must be vigilant for evidence of the PSF signature in the deconvolution.

Deconvolutions of the NGC 1068 image (Fig. 1) are reproduced in Figures 2 and 3 (Plates L6 and L7) at two different stages of processing, 85 and 341 iterations, respectively. The scaling is again logarithmic as in Figure 1, but the intensity levels have been normalized to the same data maximum in all three pictures. The inset in the lower left-hand corner shows the PSF, also with logarithmic scaling. We see no evidence of the PSF signature in the deconvolutions and consider the general character of the nuclear concentration and surrounding cloudlike structure to be valid. The light feature seen at about P.A. $300^{\circ}$ with respect to the nucleus results from an inadequately corrected flat-field anomoly. Several weak cosmic-ray events remain but are easily recognized by their sharpness.

\section{DISCUSSION AND CONCLUSIONS}

Although the image of the outer parts of the galaxy is not without value, only the nuclear region is well exposed, and only that region will be discussed here. In comparing the deconvolved images with the speckle reconstruction at similar continuum wavelengths by Ebstein et al. (1989), it is clear that the present material takes resolution of the inner structure a step further. In simplest terms, we can clearly distinguish two separate new features: an irregular cloudlike structure and a "central" knot of significantly higher surface brightness. Our discussion will be focused on these two features.

Isophotal plots of $90 \times 90$ pixel submatrices of the original image and of a 256 iteration deconvolution are shown in Figures 4 and 5 and provide some quantitative access to the data. In arbitrary units, the contours in Figure 4 begin at 1200 and occur at 900 unit intervals. The contour levels in Figure 5 correspond to twice the data values of those in Figure 4. The zero point is sky background, not galaxy background. North is

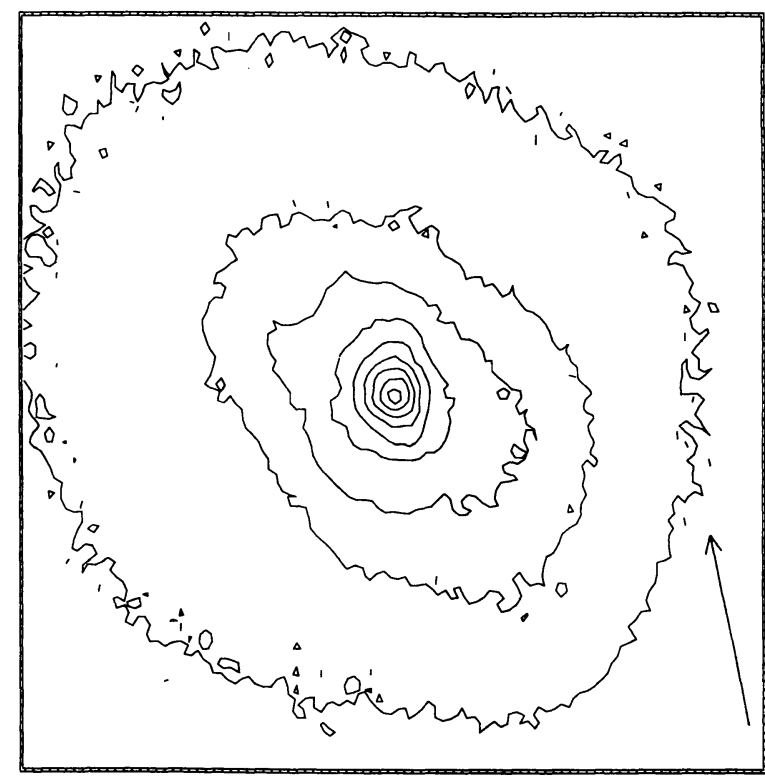

Fig. 4.-An isophotal plot of a 90 pixel square submatrix of the original image of NGC 1068 in the continuum at $5470 \AA$. The direction of north is indicated by an arrow $1^{\prime \prime}$ in length. The contour interval is 900 units beginning at 1200 units. 
FIG. 1.-A $180 \mathrm{~s}$ exposure of the nuclear region of NGC 1068 in continuum light ( $5470 \AA$ ). The displayed area is a 512 pixel square submatrix of a Planetary Camera frame. The direction of north is shown by an arrow that is $5 "$ in length.

LYNDS et al. (see 369, L32) 


\section{PLATE L6}

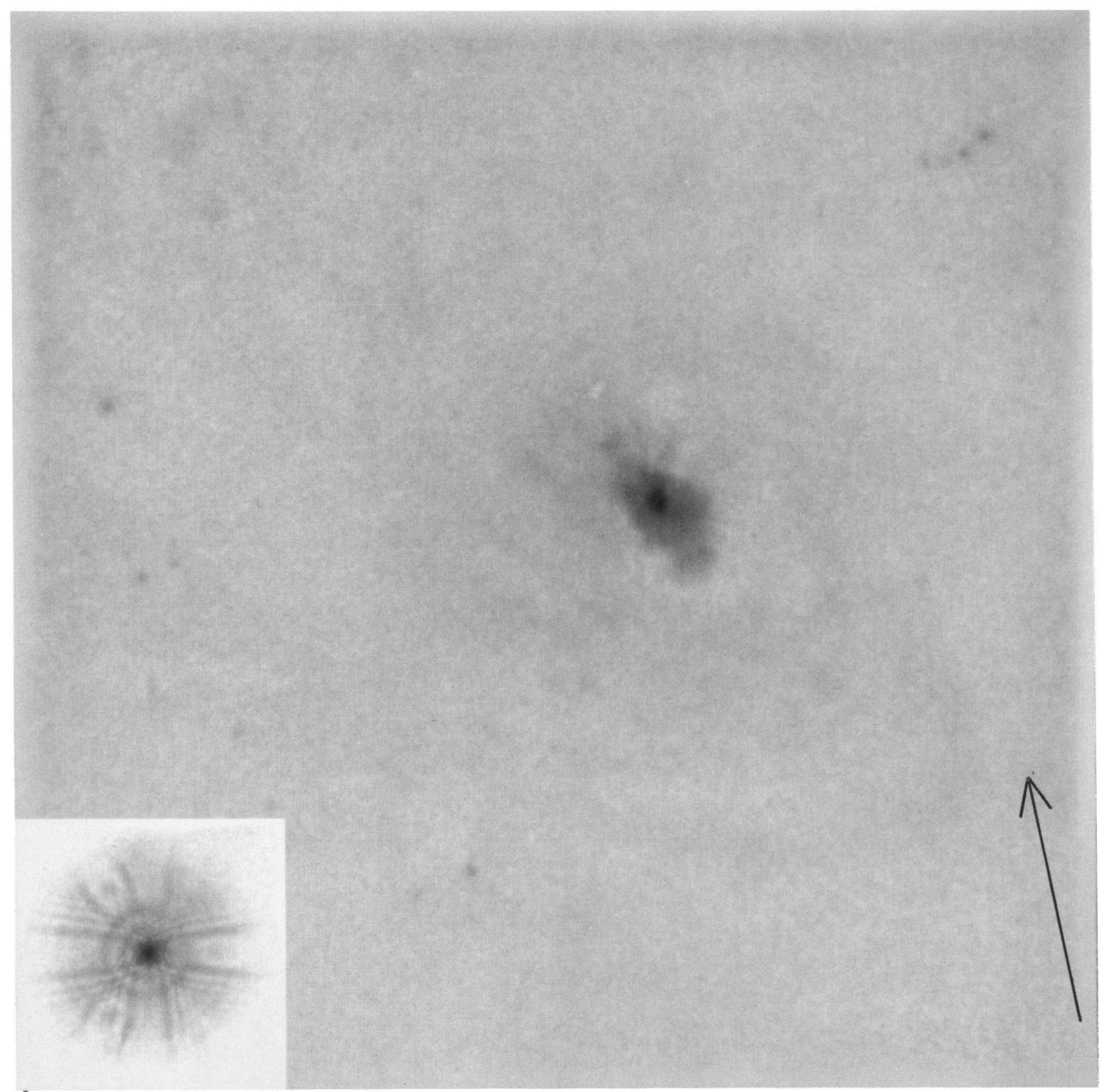

Fig. 2.-NGC 1068. The same image as in Fig. 1 after 85 deconvolution iterations. Scale and orientation are the same as in Fig. 1. LYNDS et al. (see 369, L32) 


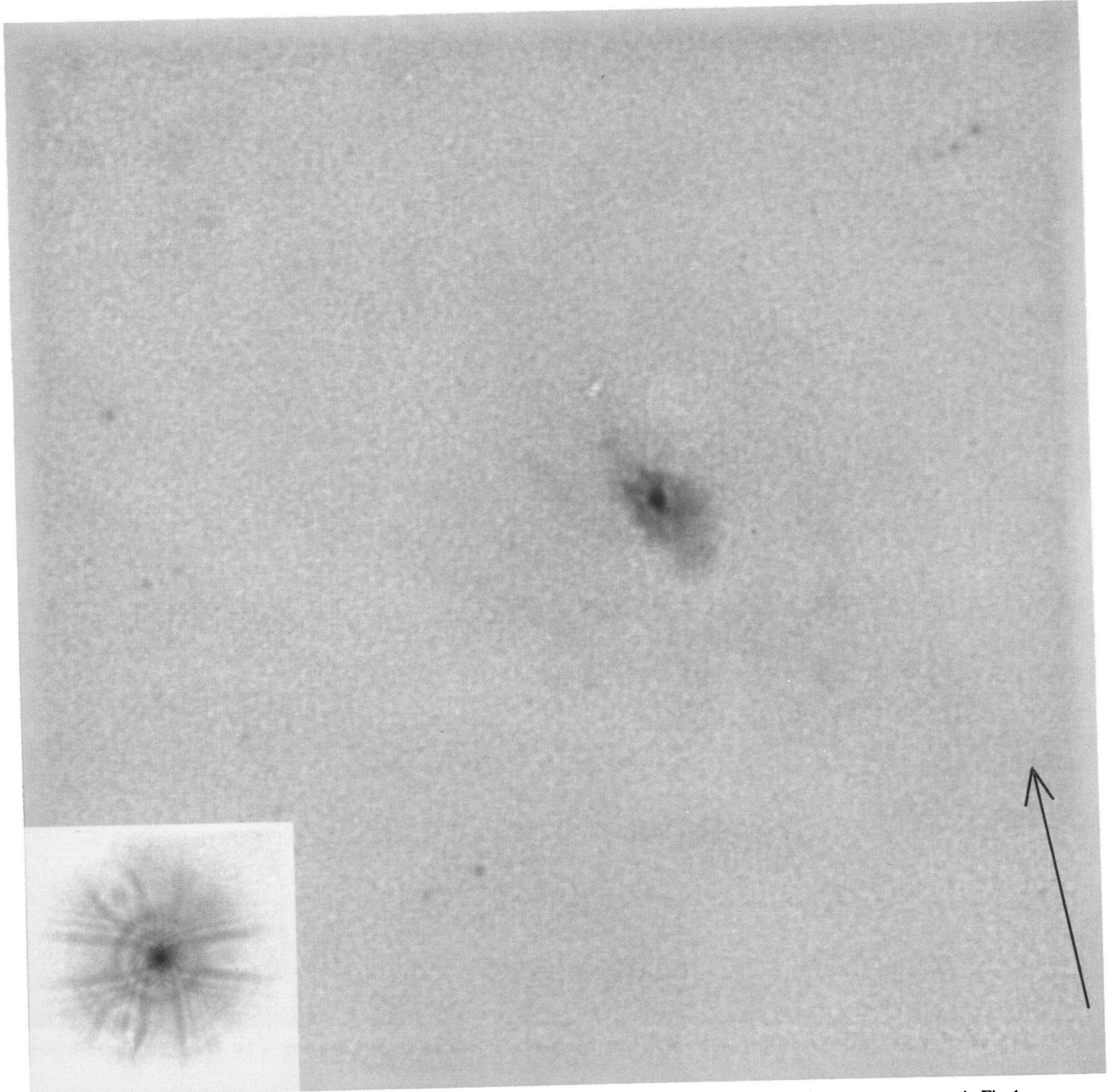

FIG. 3.-NGC 1068. The same image as in Fig. 1 after 341 deconvolution iterations. Scale and orientation are the same as in Fig. 1. LYNDS et al. (see 369, L32) 


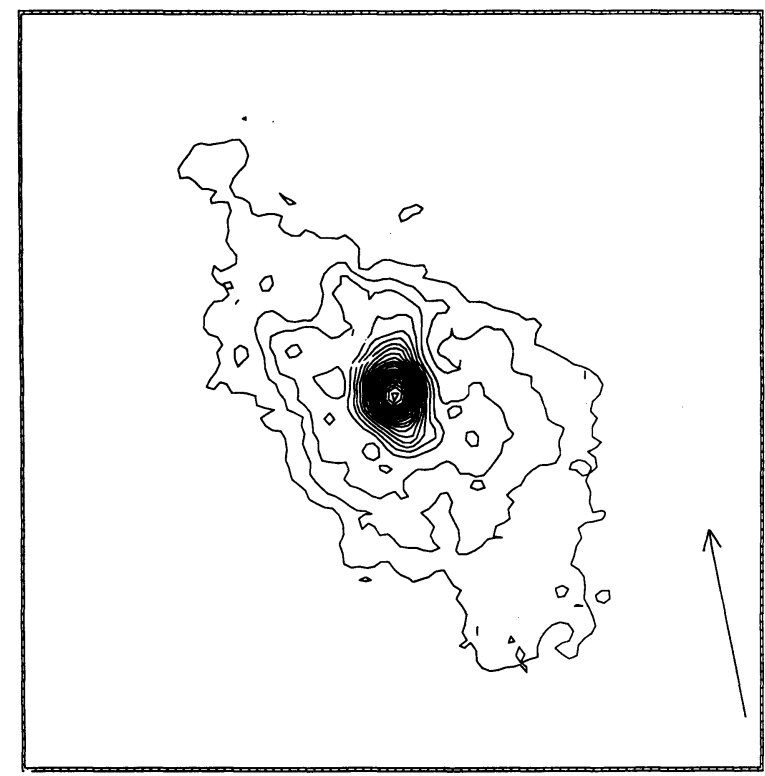

Fig. 5.-An isophotal plot similar to Fig. 4 but for a deconvolved image (256 iterations). The contour interval is 1800 units beginning at 2400 units.

the same direction as in Figures $1-3$ and is indicated by an arrow 1 " in length. Crude surface photometry indicates that $80 \%$ of the light within the 2400 unit contour of Figure 5 is attributable to the cloudlike structure and $20 \%$ to the "nuclear" feature. It is interesting that most of the light, formerly unresolved, is contributed by the relatively large cloud, now well resolved, and not by the nuclear concentration.

It is best to clarify that although we refer to the feature exhibiting the maximum surface brightness as the nucleus, we insist that this nomenclature carry no connotation other than that already stated. We do not know that this feature is the nucleus of the galaxy, whatever that may imply. Until accurate astrometry is accomplished we do not know how this "nucleus" may correspond with radio maps and the speckle reconstructions. We will, however, be making the simplest assumption for much of our discussion that this nucleus corresponds in position with the central radio component resolved by Ulvestad, Neff, \& Wilson (1987) and others, and with the bright component in the speckle reconstructions of Ebstein et al. (1989).

In analyzing the nucleus it is clear that it is broad or, as some might say, resolved, both in the original image and in the deconvolution. In the deconvolution iterations the FWHM of the nuclear feature appears to be converging on a value no smaller than $\sim 0$ ". 15 , and the profile stays distinctly blunt at the top. But the question arises as to whether or not the image could be decomposed into a point source and some remainder distribution. The answer is yes. The hypothetical point source could be strong enough even to put a depression in the remainder, down to the level of the cloud or the galaxy background, or even to sky background; and that interpretation of the image might in fact correspond to reality. But there is absolutely no evidence to suggest such an interpretation, even though it may be correct.

In discussing the cloudlike feature we first compare it to the highest resolution ground-based observations in the continuum: the speckle reconstructions of Ebstein et al. (1989). These results show an asymmetrical extension to the southwest with respect to the peak of the distribution, assumed here to correspond roughly in position with our nucleus. That interpretation is in qualitative agreement with the net asymmetry in our cloudlike feature. However, the speckle reconstruction shows little evidence of further resolution.

The best reconstruction by Ebstein et al. appears to be the one using CTIO $4 \mathrm{~m}$ observations in the light of [O III] $\lambda 5007$. They obtain four or more sources, including a very bright source which, together with two satellite sources to the SSW and NE, form a geometrical group similar to the "bent" triple source well-documented by Ulvestad et al. at 1.3 and $2.0 \mathrm{~cm}$. The separations of the emission-line sources, however, seem to be distinctly larger than for the radio components. In attempting a comparison between the pattern of emission-line sources and our continuum cloudlike structure, we find little correspondence.

Within the context of comparing our image of NGC 1068 with other high-resolution observations, at least one specific feature should be mentioned. In Figure 3 the nucleus is seen to possess a companion $\sim 0$ ".22 to the south. These two features correspond well in orientation with the central source and southern satellite source in the 1.3 and $2.0 \mathrm{~cm}$ radio maps. But the separation in the radio maps is 0.30 , and there does not appear to be any evidence in our image for a feature corresponding to the northeast radio component. In any case, we are uneasy about stating that there is a relationship, because of the real possibility that the $H S T$ suffered tracking problems during the exposure. An investigation of the images of three faint stars lying within the fields of the four Planetary Cameras failed to show evidence for spurious satellite features (or, for that matter, any broadening of the images that might vitiate our conclusion that the nuclear feature is resolved). However, it would be unwarranted to conclude that there were no tracking problems.

Taking the cloudlike structure as a whole, we feel that the appearance is not compatible with what we would expect for a dynamically relaxed system of old-population stars. The distribution could be compatible with adjoint associations of young stars, but we are in such a case puzzled by the near absence of any morphological correspondence with emissionline speckle reconstructions. One might easily get the impression that there is a small underlying source that is illuminating a cloud of scattering particles, electrons, or dust, and that is also dimly and diffusely seen as the nuclear feature in our image. However, that possibility seems also be be ruled out by the lack of correspondence between the continuum cloud and the emission-line structures revealed by speckle interferometry.

We note that most of the cloud must have been included within the 2".8 diameter entrance aperture used by Miller \& Antonucci (1983) and Antonucci \& Miller (1985) in their spectropolarimetric investigations and must have contributed most of the light. The cloud that we observe has a backgroundsubtracted average surface brightness about a factor of 8 greater than that of the underlying galaxy. Therefore, the cloud must be the major contributor to the amount, more than $83 \%$ at $5470 \AA$, of the continuum light which they determined was stellar in origin and essentially unpolarized. Thus, the possibility that the cloud is emitting synchrotron radiation appears to be definitely ruled out, and it seems an almost inescapable conclusion that the cloud we have resolved is in large measure an association of stars - stars sufficiently evolved to explain the lack of relationship with the emission-line speckle images.

In conclusion, it would seem that the most important feature 
I of the new continuum image is the lack of any sizable unreis solved nuclear point source. Current models for AGN broad, line emission conventionally involve emitting regions of order a few tenths of a parsec in size, which would appear pointlike $\stackrel{I r \mid}{\rightarrow}$ in $H S T$ if viewed directly. If such models are to be retained, we require that the broad-line region in NGC 1068 be seen only indirectly, in scattered light. One specific possibility is the torus model of Antonucci \& Miller (1985), in which a relatively clear region at the pole of an obscuring torus contains electrons that scatter broad-line region light obliquely to the viewer. At high spatial resolution, such a scattering region might plausibly look like the nuclear hot spot seen in NGC 1068, an identification that is reinforced by the near equality of the light emitted by the hot spot-some $20 \%$ of the total light within 3 - - and the fraction of light seen in the polarized continuum-also $\sim 20 \%$ within the same region.

While suggestive, this interpretation is not yet unique, as the present image does not allow us to say precisely where the polarized light is coming from. It will therefore be important to show directly, using future $H S T$ imaging and spectroscopy, whether the polarized broad-line component originates solely in the nucleus or is more diffusely spread throughout the surrounding cloud.

This research was supported in part by NASA contract NAS5-25421.

\section{REFERENCES}

Antonucci, R. R. J., \& Miller, J. S. 1985, ApJ, 297, 621

Ebstein, S. M. Carleton, N. P. \& Papaliolios, C. 1989, ApJ, 336, 103

Griffiths, R. 1990, Wide-Field and Planetary Camera Instrument Handbook, Version 2.1 (Baltimore: STScI)

Lynds, R. 1991, in preparation
Miller, J. S., \& Antonucci, R. R. J. 1983, ApJ, 271, L7

Ulvestad, J. S., Neff, S. G., \& Wilson, A. S. 1987, AJ, 93, 22

van der Hulst, J. M., Hummel, E., \& Dickey, J. M. 1982, ApJ, 261, L59

Walker, M. F. 1968, ApJ, 151, 71

Wilson, A. S., \& Ulvestad, J. S. 1983, ApJ, 275, 8 